\title{
DYNAMICAL STUDY OF THE LMC GLOBULAR CLUSTER NGC 1978 FROM CORE VELOCITY DISPERSION
}

\author{
G. MEYLAN \\ Space Telescope Science Institute \\ 3700 San Martin Drive \\ Baltimore MD 21218 \\ USA \\ P. DUBATH, M. MAYOR \\ Observatoire de Genève \\ CH-1290 Sauverny \\ Switzerland
}

\begin{abstract}
The projected velocity dispersion in the core of the Large Magellanic Cloud (LMC) intermediate-age globular cluster NGC 1978 is deduced from integrated light spectra. A numerical crosscorrelation technique gives a projected velocity dispersion $\sigma_{p}($ core $)=5.8 \pm 1.2 \mathrm{~km} \mathrm{~s}^{-1}$. Multimass anisotropic King-Michie dynamical models are applied to the observational constraints given by the surface brightness profile and the above central projected velocity dispersion. Depending on the model, the values obtained for the total mass of the cluster range from 0.36 to $1.4410^{6} M_{\odot}$, corresponding to mass-to-light ratios $M / L_{\nu}$ ranging from 1.2 to $4.2\left(M / L_{v}\right)_{\Theta}$, values typical of galactic globular clusters.
\end{abstract}

\section{Integrated light spectra}

Integrated light spectra were obtained at the European Southern Observatory at La Silla, Chile, with CASPEC, the Cassegrain Echelle Spectrograph, mounted on the ESO 3.6-m telescope. During the exposure of the globular cluster, scanning of the nucleus was done with the entrance slit, in order to cover a zone of $6 \times 6 \operatorname{arcsec}^{2}$, avoiding any problem of sampling which could occur when integrating only over a few bright stars.

\section{Numerical cross-correlation}

The new software developed for numerical cross-correlation technique (Dubathet al. 1990) mimics the photoelectric spectrometer CORAVEL (Baranne et al. 1979): it reproduces numerically with registered spectra what is achieved optically online at the telescope with CORAVEL. To take advantage of our experience with CORAVEL, only the spectral domain common to the CORAVEL

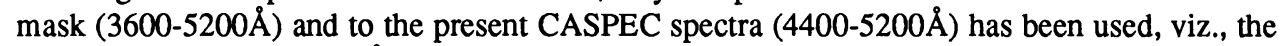
interval from 4400 to $5200 \AA$. After normalization of the cross-correlation function of the cluster, in order to have the same depth as the cross-correlation function of the two comparison stars, the projected velocity dispersion in the core of NGC 1978 is derived: $\sigma_{p}$ (core) $=5.8 \pm 1.2 \mathrm{~km} \mathrm{~s}^{-1}$. A 
discussion of the uncertainties in this projected velocity dispersion measurement is given in Dubath et al. (1990).

\section{The model}

King-Michie dynamical models are constructed in an approach identical to that of Gunn and Griffin (1979). To mimic a real cluster, heavy remnants (such as neutron stars), white dwarfs and main sequence stars are distributed into ten different subpopulations, each having the energy-angular momentum $(E, J)$ distribution function $f_{i}(E, J) \alpha\left[\exp \left(-A_{i} E\right)-1\right] \exp \left(-\beta J^{2}\right)$. In the cluster centre, thermal equilibrium is assumed to force $A_{i}$ to be proportional to the mean mass of the stars in the subpopulation considered. A model is specified by a mass function exponent $x$, and by four parameters: the scale radius $r_{c}$, the scale velocity $v_{s}$, the central value of the gravitational potential $W_{o}$, and the anisotropy radius $r_{a}$.

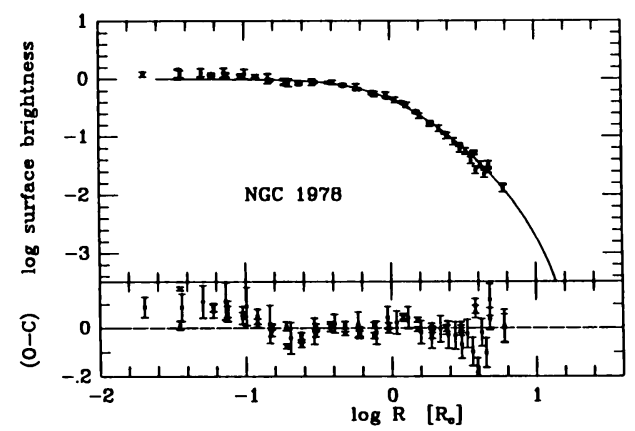

Figure 1. Observed and computed surface brightness profile for an isotropic model with $x=1.25$ and $W_{o}=8.0$. The observed profile is a merger of Mateo (1987) and Meylan and Djorgovski (in prep.) profiles.

\section{Results}

A grid of models has been calculated for a wide range of values of each parameter. The surface brightness profile lacking star counts in its outer parts and the velocity dispersion profile reduced to its core value do not fully constrain the King-Michie model. Nevertheless, areas in the parameter space are delineated. The mean value of the exponent $x$ of the mass function is $\simeq 1.00-1.50$, a value close to Salpeter's value of 1.35. Depending on the model, the values obtained for the total mass of the cluster range from 0.36 to $1.44 \times 10^{6} M_{\odot}$, corresponding to mass-to-light ratios $M / L_{v}$ ranging from 1.2 to $4.2\left(M / L_{\nu}\right)_{\ominus}$, values typical of galactic globular clusters. These results are consistent with Illingworth's (1973) method which gives $M_{t o t}=0.51 \times 10^{6} M_{\mathrm{e}}$ with $M / L_{\nu}=2.1$.

\section{References}

Baranne, A., Mayor, M., Poncet, J.L. (1979), Vistas in Astronomy 23, 279.

Dubath, P., Meylan, G., Mayor, M. (1990), Astron. Astrophys. in press.

Gunn, J.E., Griffin, R.F. (1979), Astron.J. 84, 752.

Illingworth, G.D. (1973), PhD Thesis, Australian National University, Australia.

Mateo, M. (1987), PhD Thesis, University of Washington, USA. 